\title{
MicroRNA profiles of BMSCs induced into osteoblasts with osteoinductive medium
}

\author{
ZHIXIONG YAN, YONG GUO, YANG WANG, YANAN LI and JIAHUI WANG \\ College of Biotechnology, Guilin Medical University, Guilin, Guangxi 541100, P.R. China
}

Received July 31, 2017; Accepted January 3, 2018

DOI: $10.3892 / \mathrm{etm} .2018 .5723$

\begin{abstract}
MicroRNA (miRNA) plays an important role in cell differentiation and functions as a regulator. Therefore, miRNA is important in the process of bone marrow mesenchymal stem cells (BMSCs) being induced into osteoblasts. In this study, mouse BMSCs were induced with osteoinductive medium, the indices related to osteoblastic differentiation were assayed, including alkaline phosphatase, the deposit of calcium and protein levels of osteocalcin. Using miRNA microarray and reverse transcription-quantitative polymerase chain reaction analyses, differentially expressed miRNAs in the cells, which were induced with osteoinductive medium, were selected and identified. The target genes of the differentially expressed miRNAs were then predicted using bioinformatics analysis. The results revealed that osteoinductive medium promoted osteoblastic differentiation of BMSCs, and let-7c-5p, miR-181c-3p, miR-3092-3p and miR-5132-3p were identified as differentially expressed miRNAs in the cells treated with osteoinductive medium for 14 and 21 days. Certain target genes and signal pathways related to osteoblastic differentiation of the four miRNAs were predicted. These findings indicated the four differently expressed miRNAs may be potential regulators of osteoblastic differentiation, providing a basis for further study on the regulation of miRNAs in the osteogenic differentiation of BMSCs.
\end{abstract}

\section{Introduction}

Bone marrow mesenchymal stem cells (BMSCs) are multipotent cells, with a strong ability of division and proliferation (1-3). At present, there have been great advances in the research of BMSCs osteogenic differentiation (4-6), and mainly have the following several methods: i) Chemical drug

Correspondence to: Professor Yong Guo, College of Biotechnology, Guilin Medical University, 1 Zhiyuan Road, Lingui, Guilin, Guangxi 541100, P.R. China

E-mail: guoyong74@163.com

Key words: mouse bone marrow mesenchymal stem cells, MicroRNA, osteoblast differentiation, microarray, RT-qPCR, bioinformatics induction (adding proper concentration of dexamethasone, $\beta$-glycerophosphate and ascorbic acid in the culture system), which are typically used to induce BMSCs osteogenic differentiation; ii) inducted by cytokines and growth factors (such as bone morphogenetic protein-2, transforming growth factor- $\beta$ ); and iii) stimulated differentiation under physical methods (such as mechanical strain, extracorporeal shockwaves, electromagnetic fields) $(7,8)$. However, the molecular mechanism of BMSCs which were induced into osteoblasts with osteoinductive medium remains to be fully elucidated. We believe that alteration to gene expression by microRNAs plays an important role in the osteoblast differentiation.

miRNAs are a class of small non-coding RNAs, about 21 nucleotides in length, which repress gene expression at the post-transcriptional level by degrading their target mRNAs or through translational repression $(9,10)$. miRNAs regulate cell proliferation, differentiation and apoptosis, and control physiological changes, including grow th and development (9-11). Several miRNAs are also involved as key modulators of bone formation or osteoblastic differentiation (12-15).

In previous years, some miRNAs related to osteogenic differentiation have been detected and identified in BMSC cells $(16,17)$. However, the differently expressed miRNA in osteogenic differentiation of BMSCs has not been fully studied, and the mechanism of miRNA regulating osteogenic differentiation has not been fully understood, either. In the proceess of osteogenic differentiation, we hypothesized that these differently expressed miRNAs may act as a whole in regulating specific cellular functions and pathways. In the present study, mouse BMSCs were cultured with osteoinductive medium (18), which was performed to induce osteoblastic differentiation. Subsequently, miRNA microarray and RT-qPCR analyses were performed to identify the differentially expressed miRNAs of BMSCs which were induced into osteoblasts in vitro. Specific cellular functions and signal pathways related to osteoblastic differentiation of these miRNAs were predicted using bioinformatics analysis.

\section{Materials and methods}

Cell culture and application of osteoinductive medium to cultured cells. BMSCs from C57BL/6 mice were purchased from the Cyagen Biosciences, Inc. (Guangzhou, China). The culture were maintained in $\alpha$-MEM (Invitrogen; Thermo Fisher Scientific, Inc., Waltham, MA, USA) supplemented 
with $10 \%$ fetal bovine serum (FBS; Invitrogen; Thermo Fisher Scientific, Inc.) and 1\% penicillin-streptomycin (Invitrogen; Thermo Fisher Scientific, Inc.) at $37^{\circ} \mathrm{C}$ in a humidified atmosphere containing $5 \% \mathrm{CO}_{2}$. Then, the medium was changed every 3 days. For all experiments, BMSCs were seeded at the density of $2.5 \times 10^{4}$ cells $/ \mathrm{cm}^{2}$ in the cell culture dishes and cultivated until they reached $80 \%$ confluence ( 0 day). Then the cells were induced with osteoinductive medium containing $100 \mathrm{nmol} / 1$ dexamethasone, $50 \mu \mathrm{g} / \mathrm{ml}$ ascorbic acid and $10 \mathrm{mmol} / \mathrm{l} \beta$-glycerophosphate (Invitrogen; Thermo Fisher Scientific, Inc.) and the medium was changed every 3 or 4 days. Control cultures were incubated under the same conditions with normal medium ( $\alpha$-MEM supplemented with $10 \%$ FBS and $1 \%$ penicillin-streptomycin).

ALP activity assay. Following induction of BMSCs with osteoinductive medium, the BMSCs were lysed by brief sonication on ice in radioimmunoprecipitation lysis buffer (CW Biotech, Beijing, China), and the protein concentration of the cell lysates were measured using the Bichinchoninic Acid Protein Assay kit (CW Biotech). The activity of ALP in the lysates was measured with a ALP assay kit (Nanjing Jiancheng Biotechnology Co., Ltd., Nanjing, China) using a p-nitrophenyl phosphate method, according to manufacturer's instructions. A single unit of ALP activity represented $1 \mu \mathrm{mol}$ p-nitrophenyl phosphate hydrolyzed to p-nitrophenol/min, therefore the ALP activity in the proteins was expressed in $\mathrm{U} / \mathrm{g}$ protein.

$A L P$ and alizarin red-S staining. Following cultured with osteoinductive medium, the cells were washed with PBS and fixed with $4 \%$ paraformaldehyde for $20 \mathrm{~min}$ at $37^{\circ} \mathrm{C}$. The fixed cells were soaked with the ALP staining kit (Nanjing Jiancheng Biotechnology Co., Ltd.) and Alizarin red-S staining kit (Nanjing Jiancheng Biotechnology Co., Ltd.) according to the manufacturer's instructions, and were then observed under an optical microscope.

Western blotting. After the BMSCs were induced with osteoinductive medium for 21 days, extracts of the cells were harvested in lysis buffer, protein contents of the cell lysates were quantified by BCA Protein Assay kit (CW Biotech). Proteins were separated in $10 \%$ sodium dodecyl sulfate-polyacrylamide gels, transferred to nitrocellulose membranes blocked with 2.5\% BSA in Tris-buffered saline with $0.1 \%$ Tween-20, and incubated with primary antibodies, respectively. Membranes were then incubated with secondary antibodies conjugated with horseradish peroxidase (Santa Cruz Biotechnology, Inc., Dallas, TX, USA) respectively, and immunoreactive bands were detected using an enhanced chemiluminescence detection reagent (Pierce; Thermo Fisher Scientific, Inc., Waltham, MA, USA). $\beta$-actin in cell lysates was used as a loading control, data were normalized against those of corresponding optical density of $\beta$-actin.

miRNA microarray. miRNA microarray (Agilent Technologies, Inc., Santa Clara, CA, USA) was used to detect the miRNA expression levels in the BMSCs. The miRNA expression profiles of the BMSCs which were induced with osteoinductive medium were compared with the control group (cultured without osteoinductive medium). miRNAs were enriched from total RNA extracted using a mirVana miRNA Isolation Kit and labeled with a mirVana Array Labeling Kit (Ambion; Thermo Fisher Scientific, Inc.). The labeled miRNAs were used for hybridization on each miRNA microarray to determine the differential expression of miRNAs. This procedure was repeated twice. Target labeling, hybridization, imaging and data processing were performed at Phalan Biotech (Beijing, China) according to the protocols described by the manufacturer using the Mouse miRNA OneArray Microarray (Agilent Technologies, Inc.) and Sanger miRBase 19. Data were acquired using Agilent Feature Extraction software version 10.7 (Agilent Technologies, Inc.). Further data analyses were performed using GeneSpring GX 10.0 (Agilent Technologies, Inc.).

$R T-q P C R$. The expression levels of miRNAs were detected using RT-qPCR. The primers for RT-qPCR of miRNAs were provided by Guangzhou FulenGen Co., Ltd. (Guangzhou, China), the information of the primers were listed in Table I. Following cDNA synthesis using All-in-One ${ }^{\mathrm{TM}}$ miRNA First-Strand cDNA Synthesis Kit (Guangzhou FulenGen), qPCR was performed using All-in-One ${ }^{\mathrm{TM}}$ miRNA qPCR kit (Guangzhou FulenGen) according to the manufacturer's instructions. The reactions were incubated in a 96-well optical plate at $95^{\circ} \mathrm{C}$ for $10 \mathrm{~min}$, followed by 40 cycles of $10 \mathrm{sec}$ at $95^{\circ} \mathrm{C}$, $20 \mathrm{sec}$ at $60^{\circ} \mathrm{C}$ and $10 \mathrm{sec}$ at $70^{\circ} \mathrm{C}$ (annealing and extension). Expression analysis was performed in triplicate for each sample. The average value of six reference genes were used as the normalization control (U6, U68, U70, U49A, U65, U72). The miRNA expression levels were quantified using an ABI Prism 7300 Sequence Detection system (Applied Biosystems; Thermo Fisher Scientific, Inc.).

Bioinformatics analysis. As miRNAs play their biological roles through regulating target genes expression at the post-transcriptional level, target genes of the miRNAs were predicted by online softwares, including miRDB (http://www.mirdb.org/), MicroRNASeq (http://cm.jefferson. edu/rna22v2/), TargetScan (http://wwwargetscan.org), and miRanda (http://www. microrna.org/). To identify the biological functions of these genes, Gene Ontology (GO) (http://www.geneontology.org/) and Kyoto encyclopedia of genes and genomes (KEGG) (http://www.genome.jp/) enrichment analysis were performed, respectively. Molecular Functions, Biological Process and Cellular Component of Gene Ontology were included. Search for gene annotation information using Ontology Gene database (http://www.geneontology.org/). According to the gene GO annotation, statistical methods were applied to calculate $\mathrm{P}$-values, with $\mathrm{P}<0.01$ as the significant threshold. All genes in this species were used as background genes. The high frequency notes with statistical significance were obtained and normalized to the background, information of distribution and significance of GO based gene sets were obtained. Based on the KEGG database (http://www.genome.jp/), gene sets were enriched in the biological pathway associated with osteoblastic differentiation were selected.

Statistical analysis. To identify differentially expressed miRNAs among the groups, Student's t-test was performed using SPSS 18.0 (SPSS, Inc., Chicago, IL, USA). The 
Table I. Information of primers used for miRNA RT-qPCR. ${ }^{\mathrm{a}}$

\begin{tabular}{|c|c|c|c|}
\hline Name & Mature_accession & Product_ID & Primer_ID \\
\hline mmu-let-7c-5p & MIMAT0000523 & MmiRQP0006 & Mumq-0511 \\
\hline mmu-miR-6338 & MIMAT0025081 & MmiRQP2840 & Mumq-0997 \\
\hline mmu-miR-181c-3p & MIMAT0017068 & MmiRQP3102 & Mumq-0998 \\
\hline mmu-miR-690 & MIMAT0003469 & MmiRQP1118 & Mumq-0999 \\
\hline mmu-miR-9-5p & MIMAT0000142 & MmiRQP0825 & Mumq-0167 \\
\hline mmu-miR-125b-2-3p & MIMAT0004529 & MmiRQP0877 & Mumq-0607 \\
\hline mmu-miR-1b-5p & MIMAT0005835 & MmiRQP0878 & Mumq-0185 \\
\hline mmu-miR-28b & MIMAT0019354 & MmiRQP2209 & Mumq-0903 \\
\hline mmu-miR-361-3p & MIMAT0017075 & MmiRQP3108 & Mumq-1000 \\
\hline mmu-miR-1249-3p & MIMAT0010560 & MmiRQP0081 & Mumq-0633 \\
\hline mmu-miR-3473c & MIMAT0020614 & MmiRQP2534 & Mumq-0917 \\
\hline mmu-miR-3971 & MIMAT0019356 & MmiRQP2211 & Mumq-0905 \\
\hline mmu-miR-6356 & МIMAT0025099 & MmiRQP2858 & Mumq-1001 \\
\hline mmu-miR-3072-3p & MIMAT0014853 & MmiRQP1644 & Mumq-0737 \\
\hline mmu-miR-296-5p & MIMAT0000374 & MmiRQP0364 & Mumq-0077 \\
\hline mmu-miR-223-3p & MIMAT0000665 & MmiRQP0342 & Mumq-0071 \\
\hline mmu-miR-129-2-3p & MIMAT0000544 & MmiRQP0139 & Mumq-0016 \\
\hline mmu-miR-129-1-3p & MIMAT0016994 & MmiRQP0136 & Mumq-1002 \\
\hline mmu-miR-3092-3p & МIMAT0014906 & MmiRQP1742 & Mumq-0832 \\
\hline mmu-miR-5132-3p & MIMAT0022988 & MmiRQP3535 & Mumq-1003 \\
\hline mmu-miR-483-3p & MIMAT0003120 & MmiRQP1052 & Mumq-0710 \\
\hline
\end{tabular}

${ }^{a}$ Guangzhou FulenGen Co., Ltd. (Guangzhou, China). miR, microRNA; RT-qPCR, reverse transcription-quantitative polymerase chain reaction.

experiments were repeated in triplicate. Statistical significance between the groups was measured using Student's t-test; $\mathrm{P}<0.05$ was considered to indicate a statistically significant difference.

\section{Results}

Osteoinductive medium promoted osteoblastic differentiation. The effect of osteoinductive medium on the differentiation of osteoblasts was investigated via measuring various markers of osteoblastic differentiation, including ALP, calcium deposits and OCN levels (Fig. 1). Following exposure of the BMSCs to osteoinductive medium, the ALP activities in BMSCs which were induced for 7 days and 14 days were notably higher than that in control group (Fig. 1A). Protein levels of OCN were also increased in BMSCs which were induced for 21 days (Fig. 1C). In addition, the images of ALP and Alizarin red-S staining of BMSCs indicated that the osteoinductive medium increased the levels of ALP and calcium deposits in the BMSCs at indicated time (Fig. 1B and D). ALP, OCN, and calcium deposits are all markers of osteoblastic differentiation (19-22). Therefore, the osteoinductive medium promoted osteoblastic differentiation of the BMSCs.

Identification of four differently expressed miRNAs in BMSCs which were induced with osteoinductive medium. miRNA microarray was used to detect the miRNA expression levels in BMSCs. The results revealed that the miRNAs were deregulated in the induced group under the condition of ' $\mathrm{P}<0.05$ and fold-change $>1.5$, compared with control group. Among them, the expression levels of 107 miRNAs were higher and the expression levels of 40 miRNAs were lower in the group induced with osteoinductive medium compared with the non-induced control group (Fig. 2, Table II).

RT-qPCR for further validation. 21 miRNAs were selected from the chip results (the 21 miRNAs were all expressed at 14 days and 21 days, amomg the 21 miRNAs, 2 miRNAs were both expressed at 7 days), and used RT-qPCR for further validation. The results of RT-qPCR confirmed that the expression levels of mmu-let-7c-5p, mmu-miR-181c-3p, mmu-miR-3092-3p, and mmu-miR-5132-3p had the same expression trend as the microarray in the osteoinductive medium groups (Fig. 3). In the four miRNAs, mmu-let-7c-5p was differently expressed at 7, 14 and 21 days, which was testified with both miRNA microarray and RT-qPCR. The other three miRNAs were all differently expressed at 14 and 21 days (testified with both miRNA microarray and RT-qPCR). Therefore, these four miRNAs were considered to be responsive to the osteoinductive medium applied to the BMSCs.

Target genes of mmu-let-7c-5p, mmu-miR-181c-3p, mmu-miR-3092-3p and mmu-miR-5132-3p were predicted. Four online softwares (miRanda, Clip-Seq, TargetScan, and miRDB) were applied to predict target genes for the four differentially expressed miRNAs. Prediction results of the four software were further screened and sorted out in Table III (target genes predicted by any three softwares mentioned 

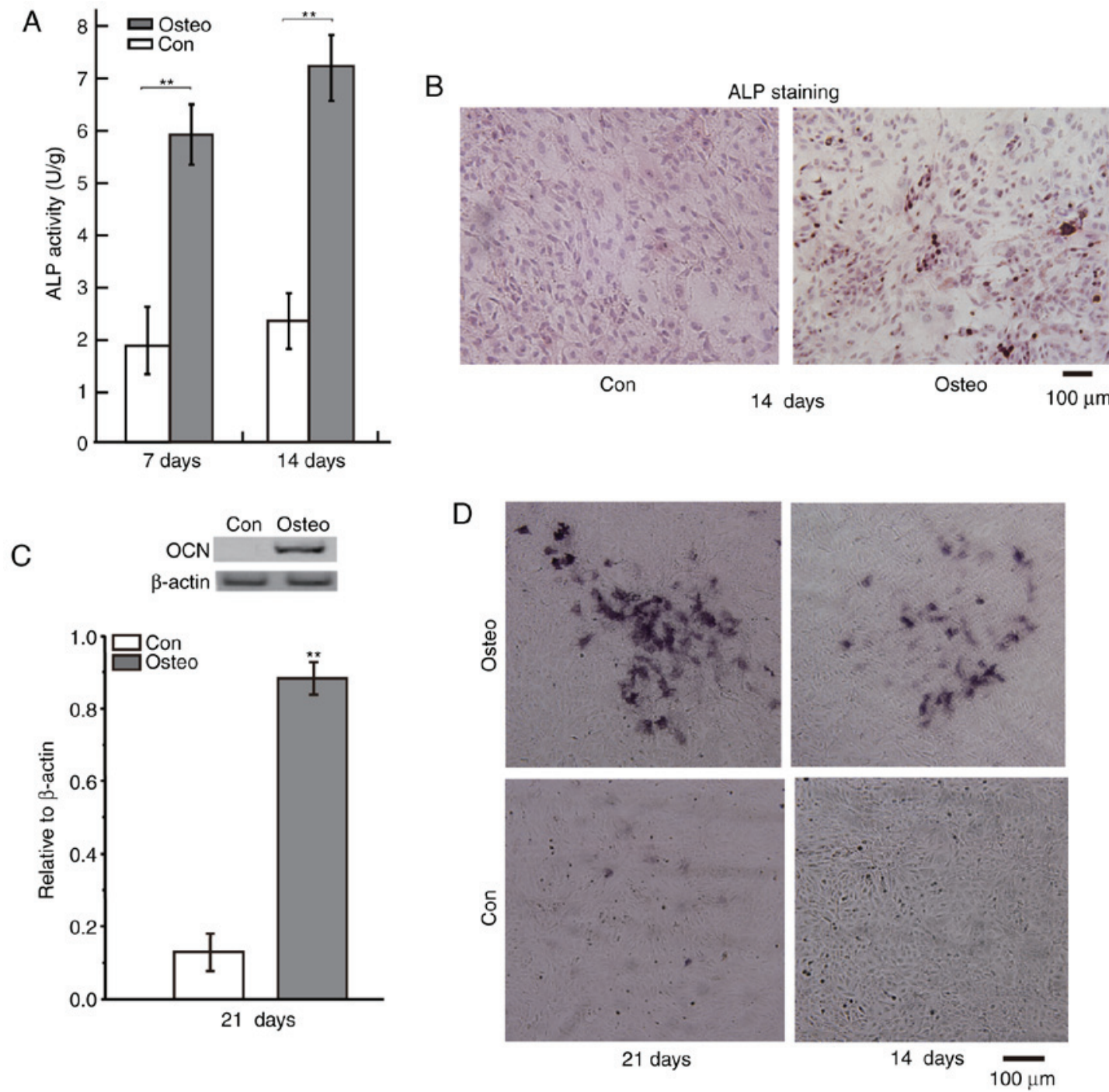

Figure 1. Following exposure of BMSCs to osteoinductive medium, ALP activity, ALP staining, protein of OCN and Alizarin red-S staining were detected at indicated time. (A) The ALP activity and (C) protein levels of OCN were notably higher. The images of (B) ALP staining and (D) Alizarin red-S staining of BMSCs indicated that the levels of ALP and calcium deposits were increased $\left(n=6,{ }^{* *} \mathrm{P}<0.01 ;\right.$ bars $\left.=100 \mu \mathrm{m}\right)$. con, control; Osteo, treated with osteoinductive medium.
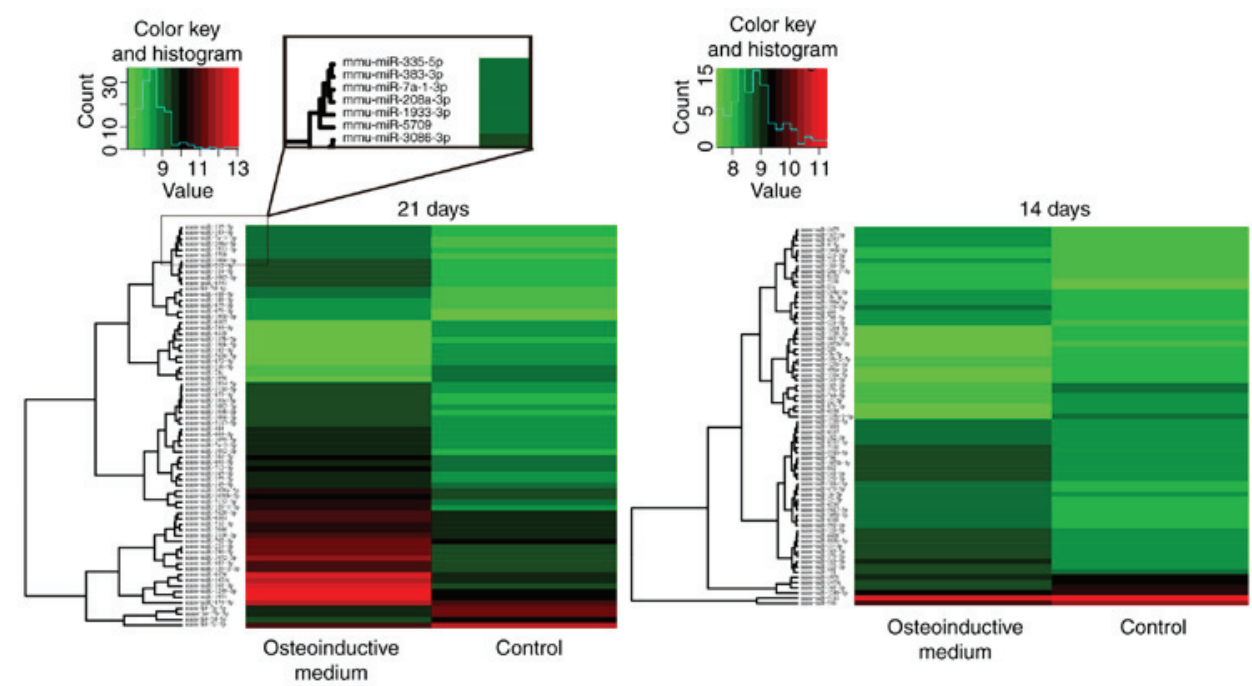

Figure 2. The result of miRNA microarray indicated that hierarchal clustering of differentially expressed microRNAs were deregulated in the induced group (14 and 21 days) under the condition of ' $\mathrm{P}<0.05$ and fold-change $>1.5$ ' compared with non-induced control group. Among them, the expression levels of 49 miRNAs were higher and the expression levels of 24 miRNAs were lower in the group induced with osteoinductive medium for 14 days compared with the non-induced control group; the expression levels of 57 miRNAs were higher and the expression levels of 15 miRNAs were lower in the group induced with osteoinductive medium for 21 days. The expression ratio represents colour ranges from green (low) to red (high), as indicated by the color key. 


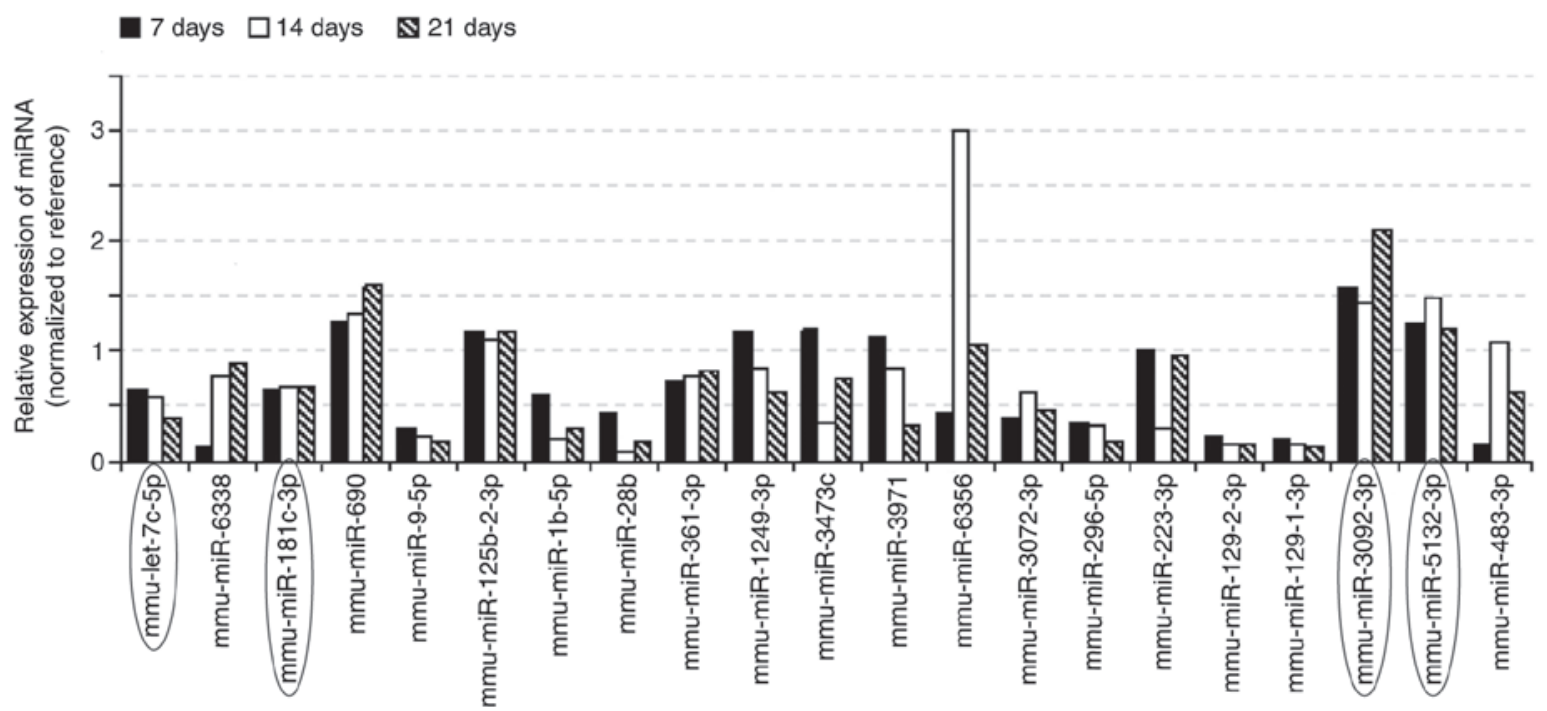

Figure 3. The results of RT-qPCR confirmed that the expression levels of mmu-let-7c-5p, mmu-miR-181c-3p, mmu-miR-3092-3p, and mmu-miR-5132-3p shared the same notable tendency in the osteoinductive medium groups as the result of miRNA microarray (7, 14 and 21 days).

Table II. miRNA microarray was used to detect the miRNA expression levels in BMSCs. The expression levels of miRNAs were upregulated or downregulated in the group induced with osteoinductive medium compared with the non-induced control group.

\begin{tabular}{lcc}
\hline Comparison & Upregulated & Downregulated \\
\hline 7 day & 1 & 1 \\
14 day & 49 & 24 \\
21 day & 57 & 15 \\
\hline
\end{tabular}

The expression levels of miRNAs were upregulated or downregulated in the group induced with osteoinductive medium compared with the non-induced control group. miRNA, microRNA.

above at the same time were regarded as a candidate target gene of corresponding miRNA). As a result, 540 genes were predicted as putative target genes of the miRNAs respond to osteoinductive medium.

Identification of the candidate target genes of miRNAs and GO/KEGG pathway enrichment analysis. To identify the biological functions of these genes, GO and pathway enrichment analysis were performed, respectively. All the GO/KEGG annotation results in pathways and molecular functions related to osteogenic differentiation or bone formation were searched to excavate functions related genes. As illustrated in Fig. 4 and Table IV, the top 20 GOs sensitive to osteoinductive medium were showed in Fig. 4. In the result of KEGG, three items with minimum P-value were selected from the statistical results of the data, showed in Table IV.

\section{Discussion}

The most basic problem in bone tissue engineering is the selection of the seed cell, which is the main source of the
Table III. Statistical table of the candidate target genes of four miRNAs predicted by the four online softwares (miRanda, Clip-Seq, TargetScan, and miRDB).

miRNA

Candidate target genes

\begin{tabular}{lr} 
mmu-let-7c-5p & 373 \\
mmu-miR-181c-3p & 30 \\
mmu-miR-3092-3p & 8 \\
mmu-miR-5132-3p & 129 \\
\hline
\end{tabular}

miRNA, microRNA.

active components of tissue engineering. At present, BMSCs are widely used in bone tissue engineering. It has the strong potential of osteogenic differentiation, the material is convenient, the self renewal ability is strong, and there is no immune rejection in transplantation $(23,24)$. Therefore, further study on the mechanism of osteogenic differentiation of BMSCs will be helpful to the clinical application of BMSCs in bone tissue engineering (25).

In recent years, researches reported that miRNA regulated osteogenesis of mesenchymal stem cells $(16,26,27)$. However, differentially expression profiles of miRNA in osteogenic differentiation of BMSCs which were induced with osteoinductive medium and the mechanism of miRNA regulating osteogenic differentiation were not fully studied yet.

In the present study, the activity of ALP, protein levels of OCN and deposit of calcium in the cell culture were all increased, which confirmed that BMSCs were induced into osteoblast with osteoinductive medium. In this study, at 7, 14 and 21 days, the differently expressed miRNAs in BMSCs treated with osteoinductive medium, were screened and testified. Four miRNAs (mmu-let-7c-5p, mmu-miR-181c-3p, mmu-miR-3092-3p, and mmu-miR-5132-3p) were all differently expressed in BMSCs treated with osteoinductive medium for 14 and 21 days, which indicated that those four miRNAs were responsive to 
Table IV. Bioinformatics analysis the main signaling pathways in BMSCs induced with osteoinductive medium.

\begin{tabular}{|c|c|c|c|}
\hline miRNA & The KEGG signaling pathway & Potential genes (total number) & (Refs.) \\
\hline \multirow[t]{3}{*}{ mmu-let-7c-5p } & MAPK signaling pathway (mmu04010) & $\begin{array}{l}\text { Casp3, Nlk, Dusp1, Nras, Map3k3, Pla2g3, } \\
\text { Map3k1, Rasgrp1, Tgfbr1, Map4k3, Map4k4 }\end{array}$ & $(11)$ \\
\hline & TGF- $\beta$ signaling pathway (mmu04350) & E2f5, Acvr1c, Gdf6, Tgfbr1, Thbs1 & $(5)$ \\
\hline & Glycerophospholipid metabolism (mmu00564) & Pla2g3, Etnk1, Lpgat1, Pld3, Pla2g15 & $(5)$ \\
\hline \multirow[t]{3}{*}{ mmu-miR-181c-3p } & Taste transduction (mmu04742) & Pde1a & (1) \\
\hline & Pertussis (mmu05133) & Sftpa1 & (1) \\
\hline & Adherens junction (mmu04520) & Pvrl3 & $(1)$ \\
\hline \multirow[t]{3}{*}{ mmu-miR-3092-3p } & $\begin{array}{l}\text { Endocrine and other factor-regulated } \\
\text { calcium reabsorption (mmu04961) }\end{array}$ & Cltc & (1) \\
\hline & Synaptic vesicle cycle (mmu04721) & Cltc & (1) \\
\hline & Bacterial invasion of epithelial cells (mmu05100) & Cltc & (1) \\
\hline \multirow[t]{3}{*}{ mmu-miR-5132-3p } & Focal adhesion (mmu04510) & Pak3, Thbs2, Igf1, Braf, tga4, Vcl & (6) \\
\hline & Regulation of actin cytoskeleton (mmu04810) & Pak3, Itgae, Itga4, Braf, Vcl & $(5)$ \\
\hline & Prion diseases (mmu05020) & C8a, Ncam1 & (2) \\
\hline
\end{tabular}

Four differentially expressed miRNAs were predicted to participate in the KEGG signaling pathways (related to osteogenic differentiation or bone formation) through regulating their potential gene targets as list. miRNA, microRNA; KEGG, Kyoto Encyclopedia of Genes and Genomes.

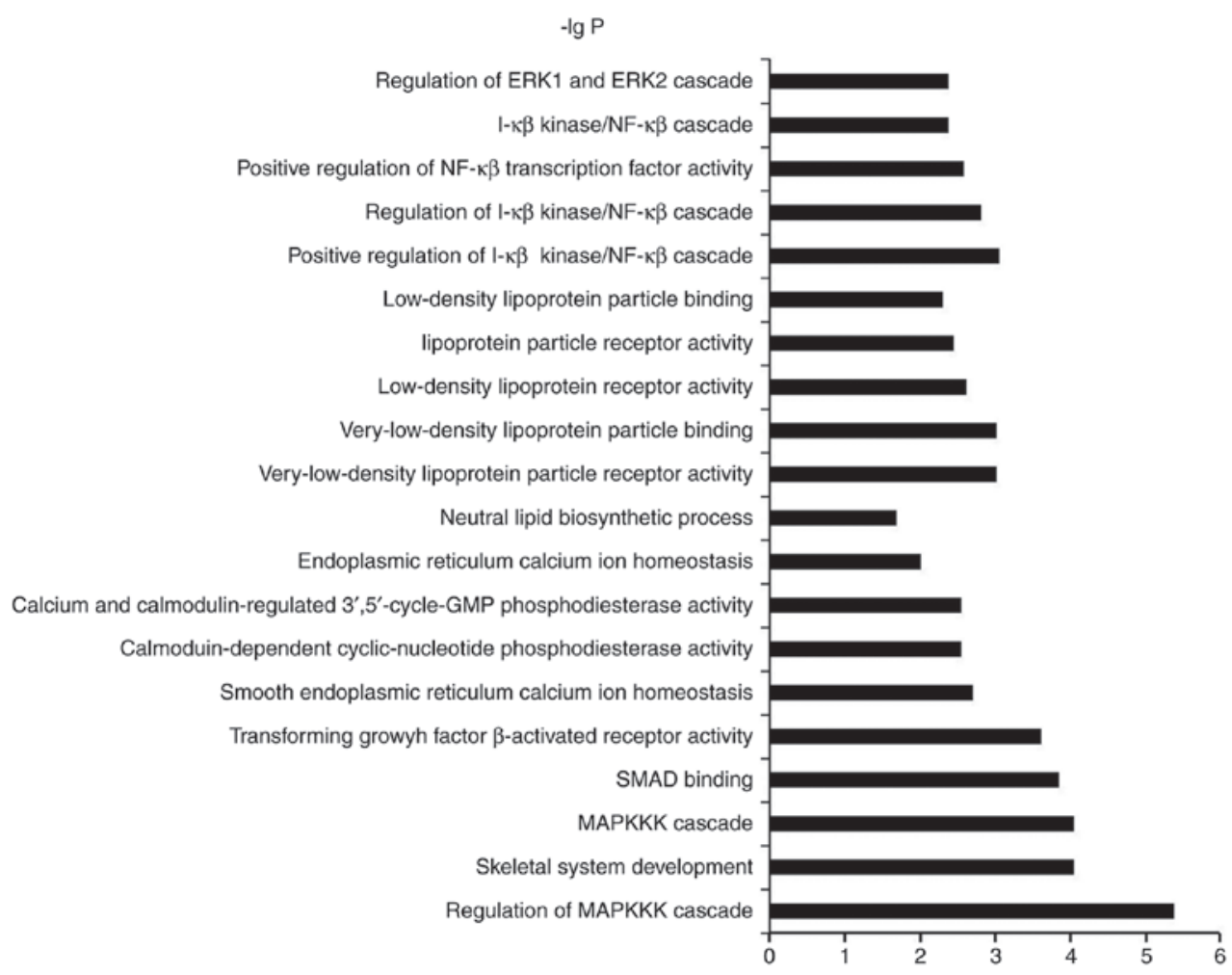

Figure 4. The top 20 significantly changed GOs of predicted target genes of the miRNAs after osteoinductive medium treatment. The y-axis showed GO category and the $\mathrm{x}$-axis showed-lgP. The larger-lgP indicated a smaller P-value.

osteoinductive medium. Bioinformatics analysis (miRanda, Clip-Seq, TargetScan, miRDB) were used to predict target genes of the four miRNAs, 540 genes were predicted as putative target genes of the four miRNAs response to osteoinductive medium.

According to the result of GO/KEGG analysis, pathways associated with osteogenic differentiation or bone formation were
TGF- $\beta$ pathway, MAPK pathway, PI-3K pathway and NF- $\kappa$ B pathway, these signaling pathways had been proved to regulate osteogenic differentiation (28-32). In addition, the predicted target genes involved in calcium metabolism, vitamin lipid metabolism and regulation of actin cytoskeleton are likely to be related to osteogenic differentiation or bone formation (33-35). 
To screen for the important genes involved in osteoblast differentiation of BMSCs, we searched potential gene targets in the Table IV, and several target genes [CASP3 (36), NLK (37), MAP3K1 (38), ACVR1c (39), GDF6 (40), PDE1a (41), IGF1 (42)] correlated with osteoblast differentiation, which have been reported. However, the other target genes require further verification. In the future, we will chose some target genes and signaling pathways which have high possibility, and validate these target genes and signaling pathways using western blot or qPCR, investigating the mechanism underlying the involvement of the miRNAs in regulating osteogenic differentiation.

In conclusion, the present study demonstrated that the osteoinductive medium promoted osteoblastic differentiation of BMSCs, and the four miRNAs (let-7c-5p, miR-181c-3p, miR-3092-3p and miR-5132-3p) were identified as differentially expressed miRNAs in the BMSCs, and were responsive to osteoinductive medium, these miRNAs are potential regulators of osteoblastic differentiation and the response of BMSCs to osteoinductive medium.

\section{Acknowledgements}

This study was supported by grants from the National Nature Science Foundation of China (nos. 11372351, 31660261, and 11432016) and the Natural Science Foundation of Guangxi (no. 2016GXNSFAA380322).

\section{References}

1. Orlic D, Kajstura J, Chimenti S, Jakoniuk I, Anderson SM, Li B, Pickel J, McKay R, Nadal-Ginard B, Bodine DM, et al: Bone marrow cells regenerate infarcted myocardium. Nature 410: 701-705, 2001

2. Sanchez-Ramos J, Song S, Cardozo-Pelaez F, Hazzi C, Stedeford T, Willing A, Freeman TB, Saporta S, Janssen W, Patel N, et al: Adult bone marrow stromal cells differentiate into neural cells in vitro. Exp Neurol 164: 247-256, 2000.

3. Friedenstein AJ, Chailakhjan RK and Lalykina KS: The development of fibroblast colonies in monolayer cultures of guinea-pig bone marrow and spleen cells. Cell Tissue Kinet 3: 393-403, 1970.

4. Liu L, Liu M, Li R, Liu H, Du L, Chen H, Zhang Y, Zhang S and Liu D: MicroRNA-503-5p inhibits stretch-induced osteogenic differentiation and bone formation. Cell Biol Int 41: 112-123, 2017.

5. Dong M, Jiao G, Liu H, Wu W, Li S, Wang Q, Xu D, Li X, Liu H and Chen Y: Biological silicon stimulates collagen type 1 and osteocalcin synthesis in human osteoblast-like cells through the BMP-2/Smad/RUNX2 signaling pathway. Biol Trace Elem Res 173: 306-315, 2016.

6. Gao M, Chen J, Lin G, Li S, Wang L, Qin A, Zhao Z, Ren L, Wang Y and Tang BZ: Long-term tracking of the osteogenic differentiation of mouse BMSCs by aggregation-induced emission nanoparticles. ACS Appl Mater Interfaces 8: 17878-17884, 2016.

7. Heino TJ and Hentunen TA: Differentiation of osteoblasts and osteocytes from mesenchymal stem cells. Curr Stem Cell Res Ther 3: 131-145, 2008.

8. Wang $\mathrm{C}$, Meng $\mathrm{H}$, Wang $\mathrm{X}$, Zhao $\mathrm{C}$, Peng $\mathrm{J}$ and Wang $\mathrm{Y}$ : Differentiation of bone marrow mesenchymal stem cells in osteoblasts and adipocytes and its role in treatment of osteoporosis. Med Sci Monit 22: 226-233, 2016.

9. Zhao Y and Srivastava D: A developmental view of microRNA function. Trends Biochem Sci 32: 189-197, 2007.

10. Bartel DP: MicroRNAs: Genomics, biogenesis, mechanism, and function. Cell 116: 281-297, 2004.

11. Stefani G and Slack FJ: Small non-coding RNAs in animal development. Nat Rev Mol Cell Biol 9: 219-230, 2008.
12. Eskildsen T, Taipaleenmäki H, Stenvang J, Abdallah BM, Ditzel N, Nossent AY, Bak M, Kauppinen S and Kassem M: MicroRNA-138 regulates osteogenic differentiation of human stromal (mesenchymal) stem cells in vivo. Proc Natl Acad Sci USA 108: 6139-6144, 2011.

13. Li Y, Fan L, Liu S, Liu W, Zhang H, Zhou T, Wu D, Yang P, Shen L, Chen J and Jin Y: The promotion of bone regeneration through positive regulation of angiogenic-osteogenic coupling using microRNA-26a. Biomaterials 34: 5048-5058, 2013.

14. Kim EJ, Kang IH, Lee JW, Jang WG and Koh JT: MiR-433 mediates ERR $\gamma$-suppressed osteoblast differentiation via direct targeting to Runx2 mRNA in C3H10T1/2 cells. Life Sci 92: 562-568, 2013.

15. Fang T, Wu Q, Zhou L, Mu S and Fu Q: miR-106b-5p and miR-17-5p suppress osteogenic differentiation by targeting Smad5 and inhibit bone formation. Exp Cell Res 347: 74-82, 2016.

16. Deng Y, Wu S, Zhou H, Bi X, Wang Y, Hu Y, Gu P and Fan X: Effects of a miR-31, Runx2, and Satb2 regulatory loop on the osteogenic differentiation of bone mesenchymal stem cells. Stem Cells Dev 22: 2278-2286, 2013.

17. Kuang W, Tan JL, Zhang HM, Duan JM, Wang WJ and Li X: miR-146a down regulates the osteogenic differentiation of murine bone marrow mesenchymal stem cells. Biomed Eng Clin Med 15: 413-417, 2011 (In Chinese).

18. Coelho MJ and Fernandes MH: Human bone cell cultures in biocompatibility testing. Part II: Effect of ascorbic acid, beta-glycerophosphate and dexamethasone on osteoblastic differentiation. Biomaterials 21: 1095-1102, 2000.

19. Wang J, Wang B, Li Y, Wang D, Lingling E, Bai Y and Liu H: High glucose inhibits osteogenic differentiation through the BMP signaling pathway in bone mesenchymal stem cells in mice. EXCLI J 12: 584-597, 2013.

20. Kim K, Dean D, Wallace J, Breithaupt R, Mikos AG and Fisher JP: The influence of stereolithographic scaffold architecture and composition on osteogenic signal expression with rat bone marrow stromal cells. Biomaterials 32: 3750-3763, 2011.

21. Liu H, Peng H, Wu Y, Zhang C, Cai Y, Xu G, Li Q, Chen X, Ji J, Zhang Y and OuYang HW: The promotion of bone regeneration by nanofibrous hydroxyapatite/chitosan scaffolds by effects on integrin-BMP/Smad signaling pathway in BMSCs. Biomaterials 34: 4404-4417, 2013.

22. Id Boufker H, Lagneaux L, Fayyad-Kazan H, Badran B, Najar M, Wiedig M, Ghanem G, Laurent G, Body JJ and Journé F: Role of farnesoid $\mathrm{X}$ receptor (FXR) in the process of differentiation of bone marrow stromal cells into osteoblasts. Bone 49: 1219-1231, 2011.

23. Gronthos S, Akintoye SO, Wang CY and Shi S: Bone marrow stromal stem cells for tissue engineering. Periodontol 2000 41: 188-195, 2006.

24. Chang SC, Chuang HL, Chen YR, Chen JK, Chung HY, Lu YL, Lin HY, Tai CL and Lou J: Ex vivo gene therapy in autologous bone marrow stromal stem cells for tissue-engineered maxillofacial bone regeneration. Gene Ther 10: 2013-2019, 2003.

25. He H, Jazdzewski K, Li W, Liyanarachchi S, Nagy R, Volinia S, Calin GA, Liu CG, Franssila K, Suster S, et al: The role of microRNA genes in papillary thyroid carcinoma. Proc Natl Acad Sci USA 102: 19075-19080, 2005.

26. Xu JF, Yang GH, Pan XH, Zhang SJ, Zhao C, Qiu BS, Gu HF, Hong JF, Cao L, Chen Y, et al: Altered MicroRNA expression profile in exosomes during osteogenic differentiation of human bone marrow-derived mesenchymal stem cells. PLoS One 9: e114627, 2014.

27. Li T, Li H, Wang Y, Li T, Fan J, Xiao K, Zhao RC and Weng X: microRNA-23a inhibits osteogenic differentiation of human bone marrow-derived mesenchymal stem cells by targeting LRP5. Int J Biochem Cell Biol 72: 55-62, 2016.

28. Liu DD, Zhang JC, Zhang Q, Wang SX and Yang MS: TGF-B/BMP signaling pathway is involved in cerium-promoted osteogenic differentiation of mesenchymal stem cells. J Cell Biochem 114: 1105-1114, 2013.

29. Yi C, Liu D, Fong CC, Zhang J and Yang M: Gold nanoparticles promote osteogenic differentiation of mesenchymal stem cells through p38 MAPK pathway. ACS Nano 4: 6439-6448, 2010.

30. Deng ZL, Sharff KA, Tang N, Song WX, Luo J, Luo X, Chen J, Bennett E, Reid R, Manning D, et al: Regulation of osteogenic differentiation during skeletal development. Front Biosci 13: 2001-2021, 2008 
31. Liu Z, Jiang H, Dong K, Liu S, Zhou W, Zhang J, Meng L, Rausch-Fan X and Xu X: Different concentrations of glucose regulate proliferation and osteogenic differentiation of osteoblasts via the PI3 kinase/Akt pathway. Implant Dent 24: 83-91, 2015.

32. Hess K, Ushmorov A, Fiedler J, Brenner RE and Wirth T: TNFalpha promotes osteogenic differentiation of human mesenchymal stem cells by triggering the NF-kappaB signaling pathway. Bone 45: 367-376, 2009.

33. Nakamura A, Dohi Y, Akahane M, Ohgushi H, Nakajima $H$, Funaoka $\mathrm{H}$ and Takakura Y: Osteocalcin secretion as an early marker of in vitro osteogenic differentiation of rat mesenchymal stem cells. Tissue Eng Part C Methods 15: 169-180, 2009.

34. Song I, Kim BS, Kim CS and Im GI: Effects of BMP-2 and vitamin D3 on the osteogenic differentiation of adipose stem cells. Biochem Biophys Res Commun 408: 126-131, 2011.

35. Rodríguez JP, González M, Ríos S and Cambiazo V: Cytoskeletal organization of human mesenchymal stem cells (MSC) changes during their osteogenic differentiation. J Cell Biochem 93: 721-731, 2004

36. You L, Gu W, Chen L, Pan L, Chen J and Peng Y: MiR-378 overexpression attenuates high glucose-suppressed osteogenic differentiation through targeting CASP3 and activating PI3K/Akt signaling pathway. Int J Clin Exp Pathol 7: 7249-7261, 2014.
37. Nifuji A, Ideno H, Ohyama Y, Takanabe R, Araki R, Abe M, Noda $M$ and Shibuya H: Nemo-like kinase (NLK) expression in osteoblastic cells and suppression of osteoblastic differentiation. Exp Cell Res 316: 1127-1136, 2010.

38. Suddason T and Gallagher E: A RING to rule them all? Insights into the Map3k1 PHD motif provide a new mechanistic understanding into the diverse roles of Map3k1. Cell Death Differ 22: $540-548,2015$

39. Tanaka K, Matsumoto E, Higashimaki Y, Katagiri T, Sugimoto T, Seino $\mathrm{S}$ and Kaji H: Role of osteoglycin in the linkage between muscle and bone. J Biol Chem 287: 11616-11628, 2012.

40. Yeh LC, Tsai AD and Lee JC: Osteogenic protein-1 (OP-1, BMP-7) induces osteoblastic cell differentiation of the pluripotent mesenchymal cell line C2C12. J Cell Biochem 87: 292-304, 2002.

41. Choi HD, Noh WC, Park JW, Lee JM and Suh JY: Analysis of gene expression during mineralization of cultured human periodontal ligament cells. J Periodontal Implant Sci 41: 30-43, 2011.

42. Saeed H, Qiu W, Li C, Flyvbjerg A, Abdallah BM and Kassem M: Telomerase activity promotes osteoblast differentiation by modulating IGF-signaling pathway. Biogerontology 16: 733-745, 2015. 